\title{
Nerve growth factor inhibits TLR3-induced inflammatory cascades in human corneal epithelial cells
}

\author{
Huiyu Chen ${ }^{\dagger}$, Jing Zhang ${ }^{\dagger}$, Yiqin Dai and Jianjiang Xu ${ }^{*}$ (D)
}

\begin{abstract}
Background: In herpes simplex epithelial keratitis, excessive TLR3-induced cellular responses after virus infection evoke inflammatory cascades that might be destructive to the host cornea. Nerve growth factor (NGF), a pluripotent neurotrophic factor with immune regulatory effect, was proved to be effective in Herpes simplex keratitis (HSK) treatment, although the detailed mechanisms remain unclear. This study aims to investigate the effects of NGF on modulating inflammatory responses triggered by TLR3 activation in human corneal epithelial cells (HCECS) in vitro.

Methods: HCECs were stimulated with TLR3 agonist, poly(l:C), in the absence or presence of NGF. Cell viability and cytotoxicity were measured by a CCK-8 assay and LDH release assay, respectively. The activation of NF-KB signaling pathway was examined using immunofluorescence staining and western blotting. Levels of proinflammatory cytokines were determined by ELISA or RT-qPCR. ROS generation and 8-OHdG positive cells were examined by a fluorometric analysis.
\end{abstract}

Results: It was shown that NGF significantly inhibited the generation of proinflammatory cytokines in HCECs triggered by TLR3 activation $(P<0.05)$, probably via suppressing NF-KB activation. NGF also impeded the upstream signal to initiate NF-KB activation by scavenging ROS by approximately $50 \%(P<0.05)$. In addition, 8-OHdG positive cells were substantially attenuated by NGF treatment $(P<0.01)$.

Conclusions: Taken together, this study indicates that NGF could inhibit TLR3-induced inflammatory cascades in HCECs, suggesting NGF as a potential therapeutic agent for HSK.

Keywords: Nerve growth factor, Toll-like receptor 3, Herpes simplex keratitis, Corneal inflammation, Oxidative injury

\section{Background}

Herpes simplex keratitis (HSK), a disorder induced by herpes simplex virus 1 (HSV-1) infection of corneal epithelial and stromal layers, is a leading cause of human blindness in developed countries [1]. Over $60 \%$ of new HSK cases present as epithelial keratitis. Cornea epithelium is the first line that protects eyes from external stimuli. It recognizes pathogen-associated molecular patterns in response to virus infection via residential Toll-like receptors (TLRs) [2], and thereby triggers innate immune responses to protect cells

\footnotetext{
* Correspondence: jianjiangxu@126.com

${ }^{\dagger}$ Huiyu Chen and Jing Zhang contributed equally to this work.

Department of Ophthalmology and Visual Science, Eye \& ENT Hospital, NHC Key Laboratory of myopia (Fudan University); Shanghai Key Laboratory of Visual Impairment and Restoration, Shanghai Medical College of Fudan University, Shanghai 200031, China
}

against microbial pathogens [3]. Although the initiated inflammatory responses are essential to clear the virus from the infected tissue, excessive TLR-induced cellular responses also evoke inflammatory cascades, which might lead to overactive inflammation infiltrating in host cornea, resulting in scar formation and vision loss $[4,5]$.

HSV-1 was shown to elicit pro-inflammatory cytokines through sequential activation of TLR2, 3, and 9 in corneal epithelial cells [6]. Among them, TLR3 has been considered as the major mediator against virus infection $[7,8]$. It recognizes double stranded RNA (dsRNA) that is generated during virus replication, or the synthetic mimic of dsRNA, named Polyinosinic-polycytidylic acid (poly (I:C)), eliciting the generation of Type I IFNs and proinflammatory factors through its downstream NF- $\mathrm{kB}$ 
pathway. Poly (I:C) is a well-recognized TLR3 agonist. Using TLR3-deficient cells and mice, Lena et al. successfully demonstrated that TLR3 conferred responsiveness and specificity to poly (I:C) [9]. Moreover, poly (I:C) could be directly bound by the extracellular domain of CD14, thereby facilitating poly (I:C) uptake and enhancing TLR3 activation [10]. Poly (I:C) triggered TLR3 activation, followed by downstream inflammatory cascades have been reported in many corneal pathologies [11-13]. Recently, emerging evidences showed that TLR3 could also trigger the generation of reactive oxygen species (ROS), an essential secondary messenger that was required for NF- $\mathrm{kB}$ activation, leading to innate immune response [14]. However, the role of ROS production during HSV-1 infection is complicated. A significant abundance of ROS could break redox homeostasis, which plays a role in chronic inflammation and tissue damage $[15,16]$. Therefore, given the pivotal role of TLR3-induced ROS generation and downstream inflammatory responses in corneal epithelium injury post virus infection, therapeutics targeting this important step may benefit in efficiently attenuating HSK progression.

Nerve growth factor (NGF) is a well-known neurotrophic factor that promotes cell growth, differentiation, survival and cell death. It is also shown to present regulatory effects in several inflammatory scenarios, including multiple sclerosis, colitis, and UV irradiation [17]. Signals mediated by NGF are propagated by its two important receptors, the highaffinity receptor TrkA and the low-affinity nonselective transmembrane glycoprotein receptor $\mathrm{p} 75^{\mathrm{NTR}}$ [18]. Recently, an increasing body of evidences demonstrated that NGF also revealed immune-modulatory effects in corneal physiopathology. Topical administration of NGF was successively applied in corneal healing [19], neurotrophic keratitis [20], immune corneal ulcer [21], dry eye syndrome [22], corneal transplantation [23], and diabetic cornea [24], highlighting the therapeutic potential of NGF in treating corneal diseases with a strong immune component involved. In HSK, endogenous NGF was shown to inhibit HSK recurrence, supported by the evidence that systemic neutralization of endogenous NGF induced HSV-1 reactivation in a rabbit model of HSK [25]. Consistently, Lambiase et al. also demonstrated that topical administration of anti-NGF antibody exacerbated disease severity in HSK rabbits [26], whilst treatment with NGF promoted a remarkable amelioration that was comparable to the effect of acyclovir [19]. Clinical treatment with NGF eye drops was shown to induce complete healing in a HSK patient that was resistant to acyclovir treatment [20], further implicating the effectiveness of NGF in HSK treatment. However, the role of NGF in HSK has not been extensively studied yet, and the detailed mechanism underlying its cytoprotective effect on HSK induced cornea epithelium injury still remains unclear.

In this study, we investigated the inhibitory effect of NGF on TLR3 triggered cellular responses, a critical step associated with HSV-1 induced cornea injury. We sought to determine whether NGF could modulate inflammatory genes and related upstream pathways in TLR3 activated human corneal epithelial cells (HCECs).

\section{Materials and methods}

Human corneal epithelial cell (HCEC) culture

As previously described [27], the immortalized HCECs were cultured in the medium consisted of 90\% DMEM/ F12, 10\% fetal bovine serum and 1\% penicillin/streptomycin in $5 \% \mathrm{CO}_{2}$ incubator at $37^{\circ}$ for $24 \mathrm{~h}$. Then HCECs were seeded in 6-, 24-, or 96-well plates at a density of $1 * 10^{5}$ cells $/ \mathrm{mL}$ adding different concentrations of poly (I: C) (Sigma p9582, USA) or NGF (Sigma SRP3015, USA) depending on the experiments. The whole research procedures were approved by the Ethics Committee of EENT Hospital of Fudan University and were performed following the declaration of Helsinki.

\section{Cell viability assay}

A CCK-8 assay (Dojindo, Japan) was used to examine cell viability according to the manufacturer's instructions. $10 \mu \mathrm{L}$ of CCK-8 solution was added to each well of the plate and the absorbance was determined at 450 $\mathrm{nm}$ using a microplate reader.

\section{LDH cytotoxicity assay}

Lactate dehydrogenase (LDH), an enzyme that released from damaged cells, is a biomarker for the integrity of cell membrane. A LDH cytotoxicity assay kit (Beyotime, China) was used to quantitatively evaluate cellular cytotoxicity according to the manufacturer's instructions. Briefly, centrifuged supernatant from treated cells was transferred into a new microplate, and then working reaction mixture was added. After incubation at room temperature for $30 \mathrm{~min}$, reactions were stopped and LDH activity was determined by spectrophotometric absorbance at $490 \mathrm{~nm}$. A whole cell lysis mixture treated by LDH release reagent (1:10 dilution, $1 \mathrm{~h}$ ) was used as a positive control.

\section{Enzyme-linked immunosorbent assay (ELISA)}

Secretion of IL-6, IL-8 and NGF were quantitated in the supernatants by commercial ELISA kits (eBioscience, CA) and read by VERSAmax 96-plate detection system (Molecular Devices, CA) with an absorbed wavelength of $450 \mathrm{~nm}$ and a reference wavelength of $570 \mathrm{~nm}$.

Intracellular reactive oxygen species (ROS) production After stimulation, HCECs were washed in PBS and treated with $200 \mu \mathrm{l}$ DMEM/F12 medium and $10 \mu \mathrm{M}$ 
Table 1 Primer Sequences for real time RT-PCR

\begin{tabular}{lll}
\hline Gene Name & Primer Pair (5'-3') & NCBI Reference sequence \\
\hline GAPDH & Fw: 5'-CGACCACTTGTCAAGCTCA-3' & NM_002046 \\
MIP1A & Rv: 5'-AGGGGAGTTCAGTGTGGTG-3' & NM_001183750.1 \\
MIP1B & Fw: 5'-TGCAACCAGTTCTCTGCATC-3' & \\
& Rv: 5'-TTTCTGGACCCACTCCTCAC-3' & NM_001018802.2 \\
IFNB & Fw: 5'-AAGCTCTGCGTGACTGTCCT-3' & \\
& Rv: 5'-GCTTGCTTCTTTGGTTGG-3' & NM_002176.3 \\
RANTES & Fw: 5'-CATTACCTGAAGGCCAAGGA-3' & NM_001278736.
\end{tabular}

H2DCF-DA (Molecular Probes, Invitrogen, 1:1000) for $30 \mathrm{~min}$ at $37^{\circ}$. H2DCF-DA probe $\left(2^{\prime}, 7^{\prime}\right.$-dichlorodihydroflulorescein diacetate), a cell-permeable fluorogenic dye, is converted by cellular esterases to non-fluorescent derivative $\mathrm{H} 2 \mathrm{DCF}$ that can be oxidized to highly fluorescent $2^{\prime}, 7^{\prime}$ - dichlorofluorescein (DCF) in the presence of ROS [28]. The plates were then read at an excitation wavelength of $480 \mathrm{~nm}$ and an emission wavelength of 530 nm (Molecular Devices, Sunnyvale, CA).

\section{Western blot analysis}

HCECs were seeded and treated in a 6-well plate, grown to $90 \%$ confluence. The main protocol was similar to that described by Zhang et al [27]. Briefly, HCECs samples were lysed in RIPA reagent (Beyotime, China) containing a protease inhibitor cocktail (Roche Diagnostics, Germany). Protein concentration in the supernatants was determined by the BCA protein assay (Beyotime, China). Protein extracts were then separated on a $10 \%$ SDS polyacrylamide gel by

\section{A}

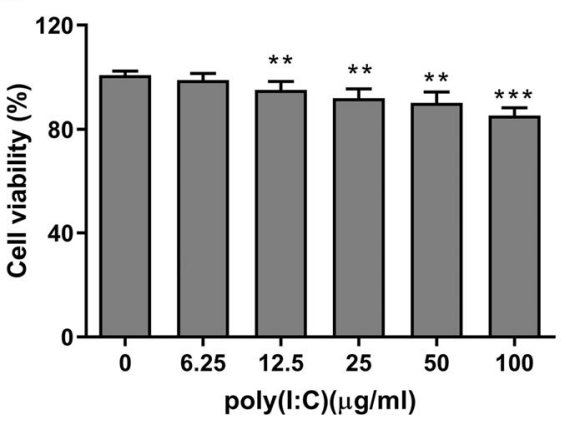

C

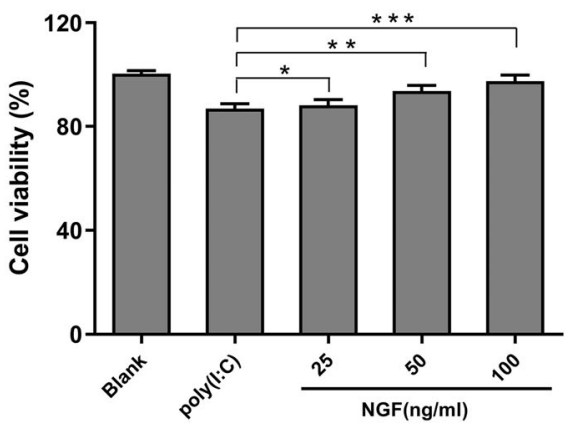

B

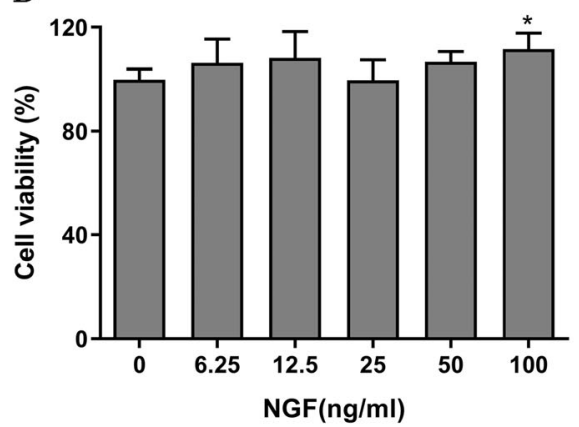

D

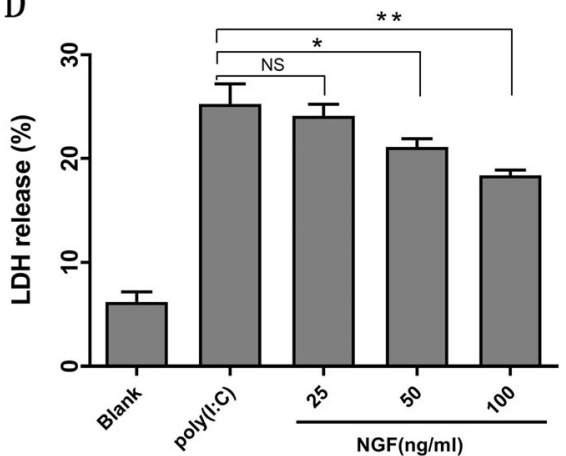

Fig. 1 NGF protected cells against TLR3-induced cytotoxicity. a-b Cell viability was determined by a CCK-8 assay in HCECs cultured with various concentrations of poly (I:C) or NGF for $24 \mathrm{~h}$. c Cell viability was determined by a CCK-8 assay in HCECs co-cultured with NGF (range from 25 ng/ $\mathrm{ml}$ to $100 \mathrm{ng} / \mathrm{ml})$ and poly (l:C) $(25 \mathrm{\mu g} / \mathrm{ml})$ for $24 \mathrm{~h}$. NGF significantly increased cell viability. $\mathbf{d}$ Cytotoxicity was determined by a LDH assay when HCECs were co-cultured with NGF (range from $25 \mathrm{ng} / \mathrm{ml}$ to $100 \mathrm{ng} / \mathrm{ml}$ ) and poly (l:C) $(25 \mathrm{\mu g} / \mathrm{ml}$ ) for $24 \mathrm{~h}$. NGF significantly suppressed LDH release in HCECs. LDH release was presented as percentage of control (whole cell lysis, 100\%). Significant differences $\left({ }^{* *} p<0.01,{ }^{* * *} p<0.001\right)$ existed compared to blank group (without poly (I:C) or NGF), conducted by one-way ANOVA and Dunnett-t test. Significant differences existed between poly $(\mathrm{I}: \mathrm{C})$ and poly $(\mathrm{l}: \mathrm{C})+\mathrm{NGF}$ treatment group, conducted by two-tailed student's $\mathrm{t}$ test. Data represented mean $\pm \mathrm{SD}$ from six independent representative experiments in each condition 
electrophoresis followed by transferring electronically to PVDF membranes. The PVDF membranes were then blocked with 5\% nonfat milk dissolved in Tris-buffered saline for $1.5 \mathrm{~h}$ at room temperature. After blocking, PVDF membranes were incubated with first antibodies against PhosphoIkB $\alpha$ (1:1000, catalog \#2859, CST), Phospho-TLR3 (Tyr759) (1:500, PA5-64722, Invitrogen), Phospho-TrkA (Tyr490) (1: 500, catalog \#4619, CST), NGF (1:500, ab52918, abcam) at $4^{\circ}$ overnight. The membranes were then washed with TBST followed by horseradish peroxidase-conjugated goat antirabbit IgG (1:1000, catalog \#7074, CST) for $1 \mathrm{~h}$ at room temperature. Chemiluminescence solution (ECL, GE
Healthcare) was used to reveal the bands, and the bands were quantified by densitometric analysis using M6EV Fluorescent and Imaging System (Peiqing, China). $\beta$-actin was used as loading control.

\section{Immunofluorescence staining}

HCECs were cultured on slides placed in 24-well plates $\left(400 \mu \mathrm{l}, 1^{*} 10^{5} \mathrm{cells} / \mathrm{ml}\right)$. Fixed in $4 \%$ paraformaldehyde for 15 min, the cell samples were permeabilized in $0.3 \%$ Triton X100 for 20 min followed by blocking with $3 \%$ fetal bovine serum dissolved in phosphate buffer saline at room temperature for $1.5 \mathrm{~h}$. After that, cell samples were incubated

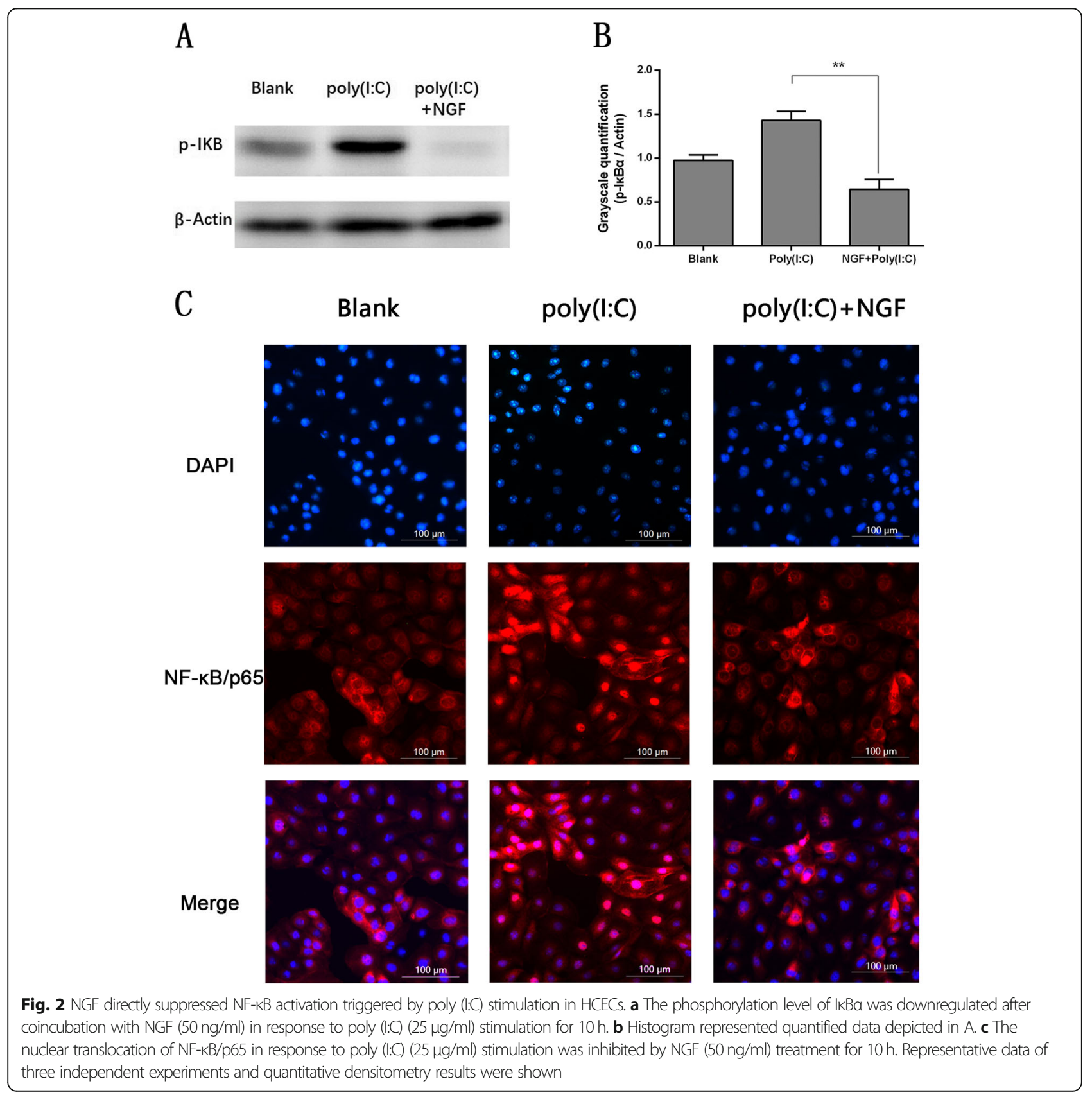


with anti-PhophoPlus NF-kB p65/RelA antibody (1:500, cata$\log \# 8214$, CST) or anti-8-Hydroxy-2'-deoxyguanosine (8OHdG) antibody (1:200, ab10802, abcam) overnight at $4^{\circ}$, followed by Alexa-Fluor 594-conjugated donkey anti-rabbit secondary antibody. The fluorescent images of the stained cell samples were obtained by a confocal microscope (Leica Microsystems, Germany).

\section{RNA extraction and quantitative real-time RT-PCR}

Total RNA was extracted using the TRIzol reagent (Invitrogen) and reverse transcribed with the PrimeScrip ${ }^{\text {Tx }}$ RT reagen Kit with gDNA Eraser (Takara RR047A; TaKaRa). Quantitative real-time RT-PCR was performed using primer sequences (Table 1) and QuantiNova SYBR Green kit (Qiagen, Germany). Target gene expression was calculated with the Comparative Ct method, shown as fold change relative to the blank group. GAPDH, a common housekeeping gene, was used as an internal control.

\section{Statistical analysis}

Results are presented as the mean \pm SD. SPSS version 18.0 (SPSS, Chicago, IL, USA) was used for statistical analysis. Statistical significance was calculated by one-way ANOVA test or the student's t-test. A $P$ value $<0.05$ were considered statistically significant.

\section{Results}

\section{Protective effect of NGF on TLR3-induced cytotoxicity in} HCECS

To explore the protective role of NGF on TLR3-induced cytoxicity, HCECs were stimulated with various concentrations of poly (I:C) in the absence or presence of NGF. Cell viability was determined by a CCK- 8 assay, as well as a LDH release assay. It was shown that poly (I:C) stimulation significantly reduced cell viability in a dosedependent manner (Fig. 1a), while NGF supplement showed protective effect especially at maximal concentration $(100 \mathrm{ng} / \mathrm{ml})(P<0.05$, Fig. $1 \mathrm{~b})$. We next tested

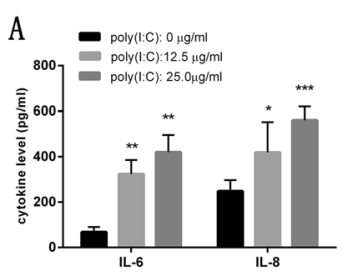

D
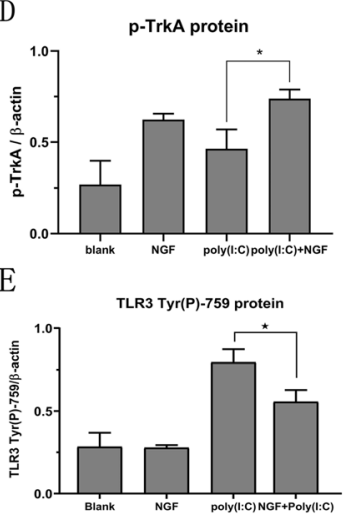

F

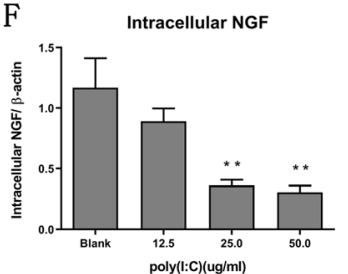

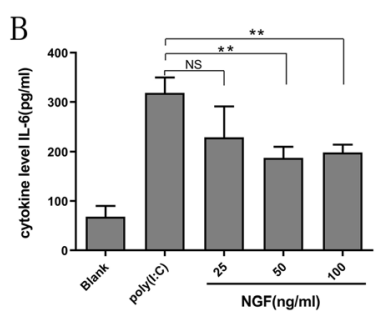

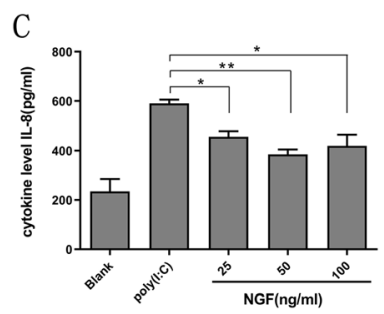

Blank NGF poly(l:C) NGF
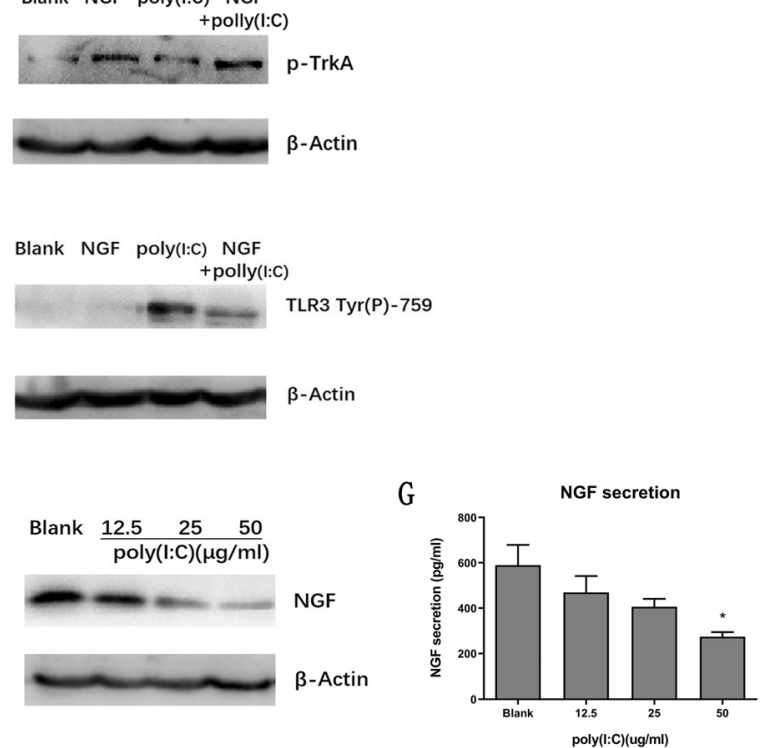

Fig. 3 NGF suppressed the production of proinflammatory cytokines in HCECs induced by TLR3 activation. a Increased secretion of IL-8 and IL-6 in HCECs stimulated with poly (I:C) for $24 \mathrm{~h}$ in a dose-dependent manner. b-c Various concentrations of NGF could suppress the secretion of IL-6 and IL-8 in HCECs treated with $25 \mathrm{\mu g} / \mathrm{ml}$ poly (l:C) for $24 \mathrm{~h}$. d TrkA phosphorylation was strongly increased after coincubation with NGF (50 ng/ml) in response to poly (l:C) $(25 \mu \mathrm{g} / \mathrm{ml})$ stimulation for $24 \mathrm{~h}$. e TLR3 phosphorylation was decreased after coincubation with NGF (50 ng/ml) in response to poly (I:C) $(25 \mu \mathrm{g} / \mathrm{ml})$ stimulation for $1 \mathrm{~h}$. f Reciprocally, TLR3 activation decreased intracellular NGF protein levels in HCECs treated with poly (l:C) for 24 h. g Stimulation with poly (I:C) for $24 \mathrm{~h}$ decreased NGF secretion by HCECS in a dose-dependent manner. Data represented mean \pm SD from three independent representative experiments in each condition 
the effect of NGF toward TLR3-induced cellular cytoxicity while HCECs were simultaneously cultured with poly (I:C) $(25 \mu \mathrm{g} / \mathrm{ml})$ and NGF (ranging from $25 \mathrm{ng} / \mathrm{ml}$ to $100 \mathrm{ng} / \mathrm{ml}$ ) for $24 \mathrm{~h}$. It was shown that NGF treatment could significantly increase cell viability (Fig. 1c) and decrease LDH release (Fig. 1d) in a dose-dependent manner, indicating the protective effect of NGF on HCECs against TLR3-induced cytotoxicity.

\section{NGF directly inhibited downstream NF-KB activation in HCECs}

NF- $\mathrm{kB}$ signaling is a critical downstream target of TLRs in inflammatory pathways through regulating the expression of a series of inflammation related genes. Once $\mathrm{I} \kappa \mathrm{B} \alpha$ is phosphorylated, it releases and activates NF-kB/p65, which transfers from the cytosol into the nucleus to regulate gene transcription [29]. Therefore, we examined whether NGF could prevent I $\mathrm{B} \alpha$ phosphorylation and subsequent nuclear translocation of NF-kB/p65. As shown in Fig. 2, poly (I:C) stimulation could significantly enhance $I \kappa B \alpha$ phosphorylation and boost the nuclear translocation of NF-kB/p65 in HCECs, while such effect was substantially inhibited by cotreatment with NGF.
NGF suppressed the production of downstream proinflammatory cytokines in HCECs induced by TLR3 activation

Next, we measured the secretion of downstream proinflammatory cytokines following TLR3 activation. In agreement with previous study [11], exposure to various concentrations of poly $(\mathrm{I}: \mathrm{C})$ remarkably increased the secretion of IL-8 and IL-6, in a dose-dependent manner (Fig. 3a). However the elevation of pro-inflammatory cytokines were significantly suppressed by NGF treatment with concentrations ranged from $25 \mathrm{ng} / \mathrm{ml}$ to $100 \mathrm{ng} / \mathrm{ml}$ (Fig. 3b, c). Notably, treatment with $100 \mathrm{ng} / \mathrm{ml}$ of NGF was found to slightly induce cell proliferation (Fig. 1b), and thereby the suppressive effect of NGF on this concentration was relatively weaker probably due to the increasing cell numbers. Therefore, HCECs were treated with NGF $(50 \mathrm{ng} / \mathrm{ml})$ and poly $(\mathrm{I}: \mathrm{C})(25 \mu \mathrm{g} / \mathrm{ml})$ to further elucidate the role of NGF on TLR3-induced cellular inflammation. The phosphorylation of NGF receptor, TrkA (Tyr-490) was significantly increased, while TLR3 (Tyr-759) phosphorylation was substantially inhibited in response to NGF treatment in TLR3 activated HCECs (Fig. 3d, e). Reciprocally, TLR3 activation was found to hamper NGF expression in a dose-dependent manner. Both intracellular NGF and secreted NGF were
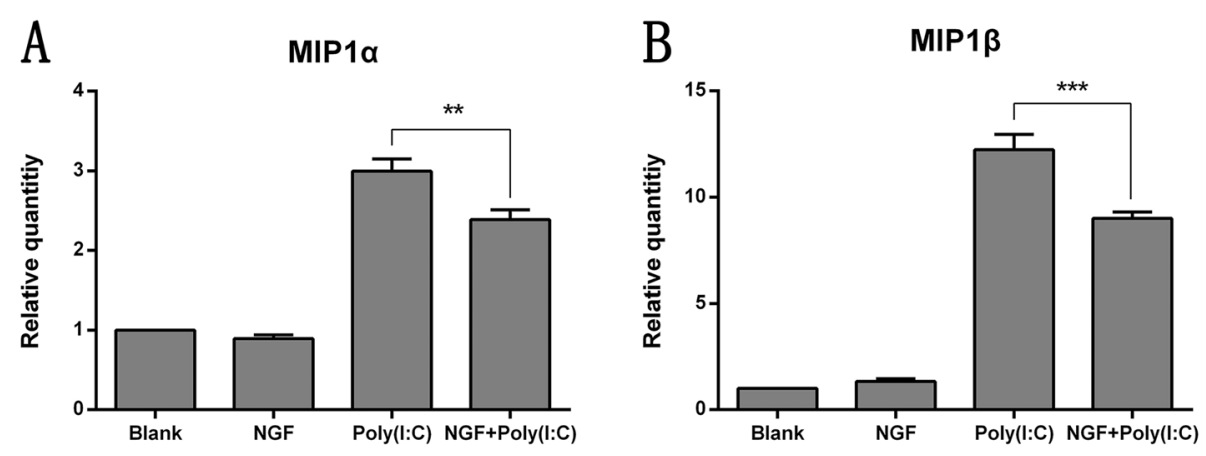

C

IFN $\beta$

D

RANTES
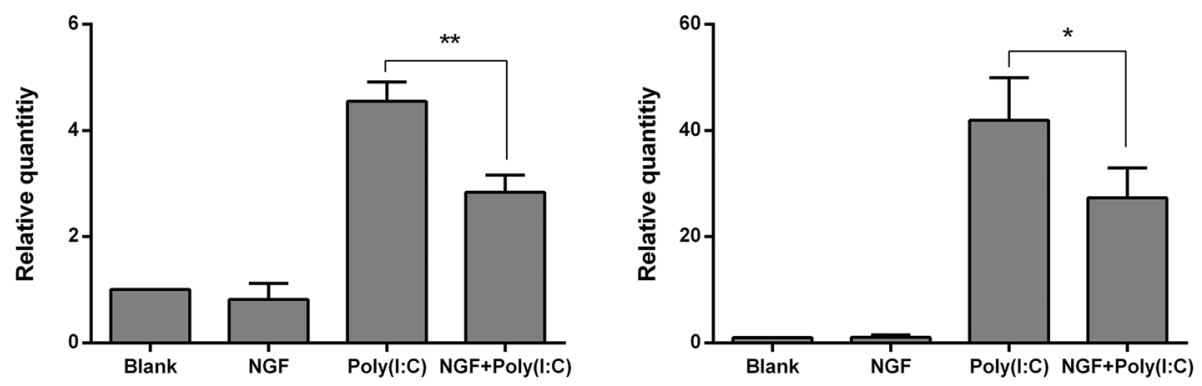

Fig. 4 NGF suppressed the expression of pro-inflammatory mediators in HCECs exposed to poly (I:C) stimulation. a-d Real time RT-PCR analysis of mRNA levels in HCECs after exposure to poly (l:C) $(25 \mathrm{\mu g} / \mathrm{ml})$ in absence or presence of NGF treatment $(50 \mathrm{ng} / \mathrm{ml})$ for $18 \mathrm{~h}$. NGF $(50 \mathrm{ng} / \mathrm{ml})$ suppressed the gene expression of pro-inflammatory mediators including IFN 3, MIP1a, MIPI $\beta$ and RANTES in HCECs treated with $25 \mu \mathrm{g} / \mathrm{ml}$ poly (I:C). Data represented mean \pm SD from three independent representative experiments in each condition 
significantly decreased upon exposure to various concentrations of poly (I:C), suggesting downregulation of NGF expression in response to TLR3-induced innate immunity (Fig. 3f, g).

It was also observed that the gene expression levels of some pro-inflammatory cytokines and chemokines including IFN $\beta, M I P 1 \alpha, M I P 1 \beta$ and RANTES were also significantly inhibited by NGF pretreatment (Fig. 4a-d).

\section{NGF attenuated TLR3-induced ROS overproduction in HCECs}

It was observed that TLR3-triggered ROS generation was required for NF- $\mathrm{kB}$ activation in monocytes. Moderate ROS generation could lead to a physical association between components of the NADPH oxidase enzyme complex, which were required for the phosphorylation and nuclear translocation [14]. In the next setting, we investigated whether NGF presented potential roles on modulating this upstream signal of initiating NF- $\mathrm{kB}$ activation. The result confirmed that poly (I:C) stimulation could promote ROS generation by HCECs, in a dosedependent manner, especially at the concentration of $25 \mu \mathrm{g} / \mathrm{ml}$ (Fig. 5a, $P<0.01$ ). However, co-incubated with NGF remarkably scavenged these overexpressed ROS by approximately $50 \%$ (Fig. 5b, c, $P<0.05$ ). Moreover, ROS can react with nucleic acids inducing single- and double-stranded DNA breaks, namely, ROS-induced 8hydroxylation of the guanine base (8-OHdG) in nuclear

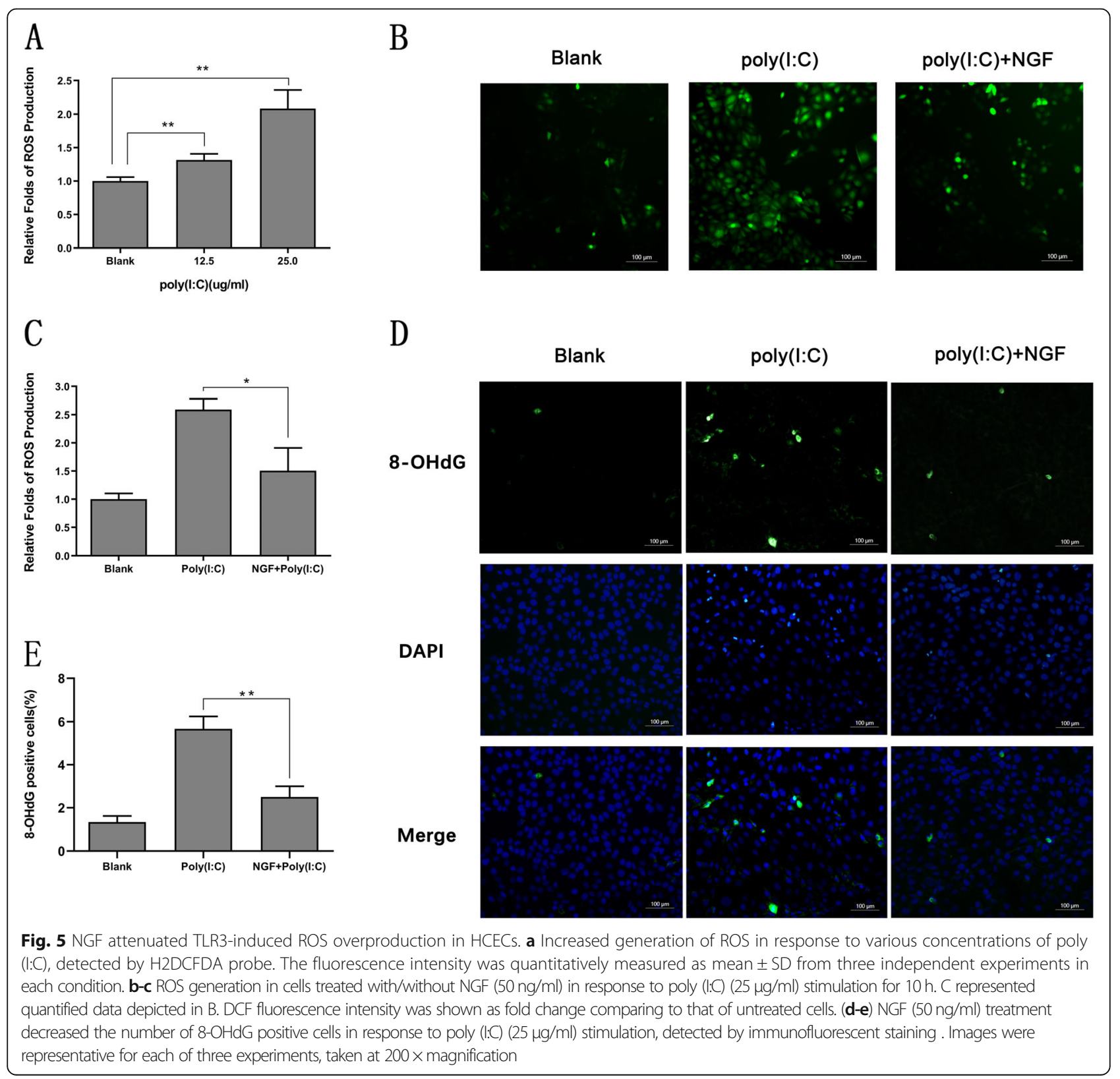


DNA [30]. We next examined the level of 8-OHdG to further investigate whether the high level of TLR3induced ROS generation led to irreversible damage to nuclear DNA. An obvious increase of 8-OHdG positive cells was observed in poly (I:C)-treated group, whilst such elevation was significantly inhibited by NGF treatment (Fig. 5d, e).

\section{Discussion}

In the current study, we triggered TLR3 activation with poly (I:C) in HCECs, mimicking one of the key steps of HSK induced corneal injury in vitro. The results demonstrated that NGF showed protective effect against TLR3induced inflammation. NGF significantly inhibited the generation of proinflammatory cytokines in HCECs that were triggered by TLR3 activation, probably via directly suppressing NF- $\mathrm{kB}$ activation. NGF also impeded the upstream signal to initiate NF- $\mathrm{KB}$ activation by scavenging ROS. Collectively, it was demonstrated firstly that NGF could ameliorate HSK associated corneal injury in vitro, highlighting NGF as a potential therapeutic agent for HSK.

Growing evidence showed that NGF played a critical role in the inflammatory response in a variety of cells. Our results showed that intracellular NGF production and secreted NGF were both decreased in response to TLR3 activation, which exacerbated abnormal secretion of inflammatory cytokines IL-6 and IL-8. In contrast, addition of NGF could attenuate it. The effect of NGF in our situation was in accordance with previous observations found in LPS-activated monocytes [31] and high glucose-treated HCECs [24] in the presence of NGF. Indeed, NGF signaling existed at the top of a hierarchical network, controlling cell cycle, Toll-like receptor, senescence regulatory pathways and VEGF signaling [32]. Besides its effect on regulating innate immunity via TLRs, NGF could promote cell cycle progression of HCECs by regulating D-type cyclins via PI3K/Akt and MAPK/Erk activation [33, 34]. NGF was also shown as a key promoter of limbal stem cells proliferation, colonyforming efficiency, and a maintainer of the LSC phenotype [32]. Moreover, the effects of NGF on promoting corneal epithelial and nerve regeneration, as well as corneal healing have been reported both in vitro and in vivo $[19,35,36]$. All these evidences underlined an important role of NGF in corneal physiopathology, and also suggested that NGF might exert therapeutic action in a wide spectrum of corneal diseases.

It was shown that HSV-1 infection quickly induced intracellular ROS that were necessary for proper activation of innate antiviral immune responses [37]. Initial generation of ROS post virus infection acts as a microbicidal compound to regulate several processes, such as expression of innate-response-related genes, NF- $\mathrm{kB}$ activation, inflammasome activation, autophagy and programmed necrosis [38-40]. However, its overproduction may exacerbate downstream inflammatory responses as well as oxidative injury depending on the absolute intracellular levels of ROS and other reactive species [16, 41]. Our results showed high levels of ROS generation and 8-OHdG, a marker of ROS-induced DNA breaks, in response to TLR3 activation. Previously, it has already been demonstrated that NGF could inhibit oxidative injury that was induced by hyperosmotic stress $[42,43]$ or high glucose levels [24] indicating the remarkable capacity of NGF on scavenging ROS generated during the pathogenesis of dry eye or diabetic cornea. Consistently, the current study also demonstrated the suppressive role of NGF on HSK related ROS generation, implicating that the underlying mechanisms of NGF on treating these three common corneal diseases might be partially overlapped.

Previously, $L i$ et al. proved that signaling pathways including NF-kB, JNK and p38 were all activated by HSV-1 infection in HCECs. Interestingly, these signaling pathways underwent two phases of activation in response to infection [44]. It was shown that HSV-1 could induce TLR7 expression at later phase after virus infection, and the induced TLR7 expression was coincident with the declined expression of TLR3, implicating that TLR3 and TLR7 might act sequentially to response to viral infection and replication in HCECs [3]. In this study, the in vitro results using TLR3 agonist-induced model in HCECs may not fully reflect the real situation in HSK. We only focused on the initial step of inflammation responses that triggered by TLR3 activation. The interplay between NGF and other HSK-related TLRs, as well as the underlying mechanisms requires in-depth investigations before its further extension to in vivo clinical conditions.

\section{Conclusion}

In summary, we demonstrated here for the first time that NGF inhibited TLR3-induced inflammatory cascades in HCECs. The present study indicated that NGF could be a promising therapeutic agent in clinical treatment of HSK.

\section{Abbreviations \\ 8-OHdG: anti-8-Hydroxy-2'-deoxyguanosine; Fw: Primer forward; GAPDH: Glyceraldehyde-3-Phosphate Dehydrogenase; HCECs: Human corneal epithelial cells; HSK: Herpes simplex keratitis; HSV-1: Herpes simplex virus 1; IFN $\beta$ : Interferon Beta; MIP1A: C-C motif chemokine ligand 3; MIP1B: C-C Motif Chemokine Ligand 4; NCBI: National Center for Biotechnology Information; NGF: Nerve growth factor; RANTES: C-C Motif Chemokine Ligand 5; ROS: Reactive oxygen species; Rv: Reverse forward; TLR3: Toll like receptor 3}

\section{Authors' contributions}

Conceived and designed the experiments: JJX, JZ, HYC, performed the experiments: HYC, analyzed the data: HYC, contributed reagents/materials/ analysis tools: JJX, YQD, wrote the paper: HYC, JZ. All authors read and approved the final manuscript. 


\section{Funding}

The authors were sponsored by the National Natural Science Foundation of China $(81670820,81700806)$. The sponsor or funding organization had no role in the design or conduct of this research.

\section{Availability of data and materials}

All data generated or analyzed during this study are included in this published article.

\section{Ethics approval and consent to participate}

All procedures were conducted according to the protocols and guidelines approved by Ethics Committee of EENT Hospital of Fudan University and were performed following the declaration of Helsinki.

\section{Consent for publication}

Not applicable.

\section{Competing interests}

The authors declare that they have no competing interests.

Received: 10 September 2019 Accepted: 18 December 2019

Published online: 26 December 2019

\section{References}

1. Al-Dujaili $L$, et al. Ocular herpes simplex virus: how are latency, reactivation, recurrent disease and therapy interrelated? Future Microbiol. 2011;6(8):877-907.

2. Lobo AM, Agelidis AM, Shukla D. Pathogenesis of herpes simplex keratitis: the host cell response and ocular surface sequelae to infection and inflammation. Ocul Surf. 2019;17(1):40-9.

3. Lambiase A, et al. Toll-like receptors in ocular surface diseases: overview and new findings. Clin Sci (Lond). 2011;120(10):441-50.

4. Paavilainen $\mathrm{H}$, et al. Innate responses to small interfering RNA pools inhibiting herpes simplex virus infection in astrocytoid and epithelial cells. Innate Immun. 2015;21(4):349-57.

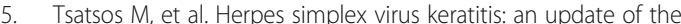
pathogenesis and current treatment with oral and topical antiviral agents. Clin Exp Ophthalmol. 2016:44(9):824-37.

6. Redfern RL, McDermott AM. Toll-like receptors in ocular surface disease. Exp Eye Res. 2010;90(6):679-87.

7. Kumar A, Zhang J, Yu FS. Toll-like receptor 3 agonist poly (I:C)-induced antiviral response in human corneal epithelial cells. Immunology. 2006: 117(1):11-21.

8. Hara Y, et al. Alteration of TLR3 pathways by glucocorticoids may be responsible for immunosusceptibility of human corneal epithelial cells to viral infections. Mol Vis. 2009;15:937-48.

9. Alexopoulou $L$, et al. Recognition of double-stranded RNA and activation of NF-kappaB by toll-like receptor 3. Nature. 2001;413(6857):732-8.

10. Lee HK, et al. Double-stranded RNA-mediated TLR3 activation is enhanced by CD14. Immunity. 2006;24(2):153-63.

11. Ueta $M$, et al. Triggering of TLR3 by polyl:C in human corneal epithelial cells to induce inflammatory cytokines. Biochem Biophys Res Commun. 2005 331(1):285-94

12. Ueta M, et al. Prostaglandin E2 suppresses poly I: C-stimulated cytokine production via EP2 and EP3 in immortalized human corneal epithelial cells. Cornea. 2012:31(11):1294-8.

13. Ye J, et al. Anti-inflammatory effects of hinokitiol on human corneal epithelial cells: an in vitro study. Eye (Lond). 2015;29(7):964-71.

14. Yang CS, et al. TLR3-triggered reactive oxygen species contribute to inflammatory responses by activating signal transducer and activator of transcription-1. J Immunol. 2013;190(12):6368-77.

15. Schachtele SJ, et al. Herpes simplex virus induces neural oxidative damage via microglial cell toll-like receptor-2. J Neuroinflammation. 2010;7:35.

16. Novo E, Parola M. The role of redox mechanisms in hepatic chronic wound healing and fibrogenesis. Fibrogenesis Tissue Repair. 2012;5(Suppl 1):S4.

17. Lambiase A, Sacchetti M, Bonini S. Nerve growth factor therapy for corneal disease. Curr Opin Ophthalmol. 2012:23(4):296-302.

18. Huang EJ, Reichardt LF. Trk receptors: roles in neuronal signal transduction. Annu Rev Biochem. 2003;72:609-42.

19. Aloe L, Tirassa P, Lambiase A. The topical application of nerve growth factor as a pharmacological tool for human corneal and skin ulcers. Pharmaco Res. 2008;57(4):253-8.
20. Aloe $L$, et al. Nerve growth factor: a focus on neuroscience and therapy. Curr Neuropharmacol. 2015:13(3):294-303.

21. Tan MH, Bryars J, Moore J. Use of nerve growth factor to treat congenital neurotrophic corneal ulceration. Cornea. 2006;25(3):352-5.

22. Lambiase $A$, et al. Alterations of tear neuromediators in dry eye disease. Arch Ophthalmol. 2011;129(8):981-6.

23. Gong N, et al. Local overexpression of nerve growth factor in rat corneal transplants improves allograft survival. Invest Ophthalmol Vis Sci. 2007:48(3): 1043-52

24. Park $\mathrm{JH}$, et al. Nerve growth factor attenuates apoptosis and inflammation in the diabetic cornea. Invest Ophthalmol Vis Sci. 2016:57(15):6767-75.

25. Hill JM, et al. Nerve growth factor antibody stimulates reactivation of ocular herpes simplex virus type 1 in latently infected rabbits. J Neuro-Oncol. 1997; 3(3):206-11.

26. Lambiase A, et al. Topical treatment with nerve growth factor in an animal model of herpetic keratitis. Graefes Arch Clin Exp Ophthalmol. 2008;246(1):121-7.

27. Zhang J, et al. Calcitriol, the Active Metabolite of Vitamin D3, Inhibits Dry Eye Related Corneal Inflammation In Vivo and In Vitro. Ocul Immunol Inflamm. 2017;27:1-9.

28. Voloboueva LA, et al. (R)-alpha-lipoic acid protects retinal pigment epithelial cells from oxidative damage. Invest Ophthalmol Vis Sci. 2005;46(11):4302-10.

29. Panwalkar A, Verstovsek S, Giles F. Nuclear factor-kappaB modulation as a therapeutic approach in hematologic malignancies. Cancer. 2004;100(8): 1578-89.

30. Halliwell B. Can oxidative DNA damage be used as a biomarker of cancer risk in humans? Problems, resolutions and preliminary results from nutritional supplementation studies. Free Radic Res. 1998;29(6):469-86.

31. Prencipe $\mathrm{G}$, et al. Nerve growth factor downregulates inflammatory response in human monocytes through TrkA. J Immunol. 2014;192(7):3345-54.

32. Kolli $\mathrm{S}$, et al. The role of nerve growth factor in maintaining proliferative capacity, Colony-forming efficiency, and the Limbal stem cell phenotype. Stem Cells. 2019;37(1):139-49.

33. Wu D, et al. MicroRNA494 inhibits nerve growth factorinduced cell proliferation by targeting cyclin D1 in human corneal epithelial cells. Mol Med Rep. 2017:16(4):4133-42

34. Hong J, et al. NGF promotes cell cycle progression by regulating D-type cyclins via PI3K/Akt and MAPK/Erk activation in human corneal epithelia cells. Mol Vis. 2012;18:758-64.

35. Di G, et al. Corneal epithelium-derived Neurotrophic factors promote nerve regeneration. Invest Ophthalmol Vis Sci. 2017;58(11):4695-702.

36. Lambiase A, et al. Nerve growth factor promotes corneal healing: structural, biochemical, and molecular analyses of rat and human corneas. Invest Ophthalmol Vis Sci. 2000;41(5):1063-9.

37. Gonzalez-Dosal R, et al. HSV infection induces production of ROS, which potentiate signaling from pattern recognition receptors: role for Sglutathionylation of TRAF3 and 6. PLoS Pathog. 2011;7(9):e1002250.

38. Matsuzawa A, et al. ROS-dependent activation of the TRAF6-ASK1-p38 pathway is selectively required for TLR4-mediated innate immunity. Nat Immunol. 2005;6(6):587-92.

39. Tal MC, et al. Absence of autophagy results in reactive oxygen speciesdependent amplification of RLR signaling. Proc Natl Acad Sci U S A. 2009; 106(8):2770-5.

40. Marino-Merlo F, et al. NF-kappaB-Dependent Production of ROS and Restriction of HSV-1 Infection in U937 Monocytic Cells. Viruses. 2019;11(5):1.

41. Grandvaux N, Soucy-Faulkner A, Fink K. Innate host defense: Nox and Duox on phox's tail. Biochimie. 2007;89(9):1113-22.

42. Kang SS, et al. Effect of nerve growth factor on the in vitro induction of apoptosis of human conjunctival epithelial cells by hyperosmolar stress. Invest Ophthalmol Vis Sci. 2014:55(1):535-41.

43. Sun $Z$, et al. NGF protects against oxygen and glucose deprivation-induced oxidative stress and apoptosis by up-regulation of HO-1 through MEK/ERK pathway. Neurosci Lett. 2017;641:8-14.

44. Li $\mathrm{H}$, et al. Herpes simplex virus 1 infection induces the expression of proinflammatory cytokines, interferons and TLR7 in human corneal epithelial cells. Immunology. 2006;117(2):167-76.

\section{Publisher's Note}

Springer Nature remains neutral with regard to jurisdictional claims in published maps and institutional affiliations. 